\title{
Channel Estimation Using EKF - ZCT Based Orthogonal STBC MIMO OFDM System
}

\author{
Sanjay Kumar KVS \\ Research Scholar, \\ Department of Electronics and Communications Engineering Jawaharlal Nehru Technological \\ University Kukatpally, Hyderabad, India \\ Dr. B. L. Malleswari \\ Principal, \\ Department of Electronics and Communications Engineering Sridevi Women's Engineering College \\ Affiliated to JNTU, Hyderabad, India
}

\begin{abstract}
Multiple-input multiple-output orthogonal frequency division multiplexing (MIMO-OFDM) transmission can improve both the transmission capacity and performance due to diversity gain. However, one of the major drawback of MIMO-OFDM have high peak-to average power ratio (PAPR) in order to the signals transmitted on different antennas. The transmitted bits must be channel-encoded as they are particularly critical to the performance of the considered OFDM system. Consequently, this channel encoding highly increases the system complexity and decreases the transmission data rate. These two problems are subjected to transmitter diversity that can overcome by incorporating orthogonal space-time block coded (OSTBC) and Zadoff-Chu Transform (ZCT) with the MIMO-OFDM. Moreover, the receiver diversity is can be improved using of Extended Kalman Filter $(E K F)$. By enhancing the transmitter diversity and receiver diversity, we can achieve the spatial diversity, which leads to reduce the BER values and PAPR values. Finally, the performance of our proposed ZCT-OSTBC-EKF methodology is evaluated based on BER and PAPR by compared with the existing methodology.
\end{abstract}

Keywords: Extended Kalman Filter, Multiple-input multiple-output, orthogonal frequency division multiplexing, orthogonal space-time block coded, Zadoff-Chu Transform.

\section{INTRODUCTION}

A communication system consists of multiple antennas for transmitting and receiving units named as MIMO. More channels that are independent can be obtained from MIMO system rather than improving the performance of traditional antennas is through single data flow. With the intention of solving the practical problems and improving the performance of MIMO, many research focused on improving the channel capacity through increasing the number of antennas linearly with specific constraints [1]. Even though, the diversity of MIMI will be reduced due to the channel correlation, which is obtained when the distance of multiple antennas become close to each. With intention of decreasing the level of channel correlation, one of the widely used methodology is orthogonal frequency division multiplexing (OFDM) is a high spectral efficiency modulation in wireless communications [2]. The fundamental thought of OFDM modulation is to grouping a wideband signal bandwidth into an arrangement of orthogonal subcarriers, and to prompt a symbol period that is any longer than the bit duration. The long stretch of OFDM symbol gives great assurance against multipath interference of channel [3]. The blend of MIMO and OFDM transmission has started a lot of exploration exercises. The MIMO-OFDM is a broadband transmission joining the MIMO framework and the OFDM modulation procedure and it can make use the spatial dimension capacity to enhance the wireless link [4].

The major problems of wireless communication using MIMO-OFDM is transmitter diversity, channel diversity, Peak-Average Power Ratio (PAPR), and receiver diversity. These problems can be solve by increasing the number of transmitter antenna and receiver antenna but it takes more cost. With the intention of solving the above problem with cost efficiency, we plan to incorporate the OSTBC, ZCT 
and Extended Kalman Filter (EKF) methodologies in our research. With the intention of combat, the channel fading in MIMO system, the widely used coding technique is space-time block codes (STBC) which can solve the diversity problem in MIMO with the aid of orthogonal STBC space-time block codes (STBC) is special (OSTBC) class of STBC. STBC MIMO-OFDM has been viewed as a promising answer for future wireless communication systems [4].

With the intention of reducing the PAPR in OFDM, the subcarriers signals are added through inverse fast Fourier transform (IFFT) operation which helps to increase the performance of high-power amplifier (HPA) at the transmitter [5, 6] [7]. There are many techniques used to reduce the PAPR of MIMO-OFDM system that are Clipping and filtering [8], Partial transient sequence (PTS) [9], Selected mapping(SLM) [10], Zadoff-Chu transform (ZCT) [11]. Zadoff-Chu sequence provides constant amplitude output signal with optimum correlation properties and reducing the cost and complexity of output signal [12]. With the aid of Extended Kalman filter, we reduce the receiver diversity problem. In practical, there is a problem to improve channel diversity with the any component in directly. We can improve channel diversity by increasing the transmitter diversity and receiver diversity.

\section{RELATED WORKS}

Xiaodong Zhu [4] proposed a clipping scheme for PAPR reduction in STBC MIMO-OFDM systems. Unlike the conventional schemes, the clipping operation was performed before STBC encoding in our scheme. Their scheme offers lower BER while maintaining the same PAPR reduction as the conventional solutions. Additionally, they derived the symbol-error rate (SER) and BER expressions for the clipped signals over multipath fading channels.

Imran Baig et al [13] presented a Zadoff-Chu matrix transform (ZCMT) based space-time-blockcoded (STBC) MIMO-OFDM system with reduced PAPR. Extensive simulations have been performed to analyse the PAPR of the proposed system with root-raised cosine (RRC) pulse shaping. Their Simulation results shown that, ZCMT precoded STBC MIMO-OFDM system has low PAPR when compared to both the Walsh-Hadamard transform (WHT) precoded STBC MIMO-OFDM systems and the conventional STBC MIMO-OFDM systems.

Javaid A. Sheikh et al [14] presented a PAPR reduction technique for OFDM based on the fusion of OSTBC encoder and Spreading code sequence and concatenating the concept of MIMO with DSCDMA. The OFDM modulator has been implemented by Inverse Fast Fourier Transform (IFFT). The result obtained from the IFFT is subjected to spreading code, which spreads, and subsequently each signal is subjected to modulation process with distinctive subcarrier with every chip. The modulated signals are passed to OSTBC that uses various number of transmit antennas for encoding process. They defined number of antennas by manually or through adaptation algorithm. They compared their proposed methodology with the conventional SLM technique in terms of PAPR and they achieved significant reduction of PAPR when the values of M-Ary phase modulations become high.

\section{Problem Statement}

Multiple-input multiple-output orthogonal frequency division multiplexing (MIMO-OFDM) transmission can improve both the transmission capacity and performance due to diversity gain. However, when the antennas are close to each other in a MIMO-OFDM system, the diversity order will be decreased because of channel correlation. With the intention of improving the performance of MIMO-OFDM in terms of accuracy of received signal with the aid of blind channel estimation technique. In this paper, we focus on major factor, which are affects the MIMO-OFDM system such as transmitter diversity and receiver diversity to enhance the accuracy of received signal.

In order to increasing the diversity gain in MIMO systems STBC has been employed through coding around multiple antennas throughout duration of multiple symbols. Alamouti STBC consist data coded across space and time in order to enhance the reliability of transmission and STBC works well with the pair of transmit and single receiver antenna. In this paper, we plan to incorporate orthogonal STBC, which simplifies Alamouti STBC to attain the maximum diversity indicated by MIMO. The combination of OFDM with MIMO helps to increase the diversity gain as well as to increase the system capacity of on wireless channel. The OFDM makes partition of wideband signal bandwidth into set of orthogonal subcarriers to a symbol for better protection versus multi-path interference of channel. The induced symbol period of OFDM has longer than the conventional bit period. The peak 
value of transmitted signals from OFDM become high in time domain when compared to average value of entire system because of various independent subcarrier components are attached via an IFFT operation. Due to this property, the high PAPR leads to nonlinear distortion of the high-poweramplifier (HPA) and results in inter-carrier-interference (ICI) plus out-of-band radiation increase the peak power at the output of the high-power amplifier (HPA) at the transmitter. The major problem of using OFDM is PAPR that will be decreased using ZCT technique. The receiver diversity can be improve using Extended Kalman Filter (EKF).

\section{Proposed ZCT based Orthogonal STBC MiMO OFDM System}

Initially, the random signal is modulated using modulation technique in this paper we incorporate BPSK modulator (need to write details about qpsk modulation). The modulated baseband symbols are converted to the parallel order through serial-to-parallel converter $(\mathrm{S} / \mathrm{P})$, which is mainly used to reduce complexity of the signal. The outcome of the S/P converter produces the complex vectors can be expressed as $S_{k}=\left[S_{1}, S_{2}, \cdots S_{L-1}, S_{L}\right]^{T}$. The complex vector is passed to OSTBC encoder to generate $\mathrm{T}$ number of complex vectors for $\mathrm{T}$ number of antennas.

$$
\begin{aligned}
& S_{1}=\left[S_{1},-S_{2}, S_{3}, S_{4} \cdots \cdots \cdots, S_{L-1},-S_{L}^{*}\right]^{T} \\
& S_{2}=\left[S_{1},-S_{4}^{*}, S_{3}, S_{2}^{*}, \cdots \cdots \cdots, S_{L}^{*}, S_{L-1}^{*}\right]^{T} \\
& S_{t}=\left[S_{1}, S_{2}, S_{4}^{*},-S_{3}^{*}, \cdots \cdots \cdots,-S_{L-1}^{*}, S_{L}\right]^{T} \\
& S_{T}=\left[S_{3}^{*}, S_{2},-S_{3}^{*}, S_{4}, \cdots \cdots \cdots, S_{L}^{*},-S_{L-1}^{*}\right]^{T}
\end{aligned}
$$

The generated sequences obtained from OSTBC are subjected to Zadoff-Chu Transform. In ZCT the matrix is generated with the size of $N=L \times L$.

\subsection{Computation of Matrix A (ZCT)}

Zadoff-Chu sequences can be defined for a sequence of size $L$ from which, the Zadoff-Chu matrix can be generated with the size of $N=L \times L$ by reshaping with $k=m+l L$.

$$
a_{n}= \begin{cases}e^{\frac{j 2 \pi r}{N}\left(\frac{k^{2}}{2}+q k\right)} & \text { for Neven } \\ e^{\frac{j 2 \pi r}{N}\left(\frac{k(k+1)}{2}+q k\right)} & \text { for Nodd }\end{cases}
$$

Where, $k=0,1,2, \cdots, N-1, q$ is any integer, and the value of $r$ is any integer relatively prime to $N$ and $j=\sqrt{-1}$. The Zadoff-Chu matrix transform is presented in following.

\subsection{ZCT on OSTBC}

$$
A=\left[\begin{array}{cccc}
a_{00} & a_{01} & \cdots & a_{0(L-1)} \\
a_{10} & a_{11} & \cdots & a_{1(L-1)} \\
\vdots & \vdots & \ddots & \vdots \\
a_{(L-1) 0} & a_{(L-1) 1} & \cdots & a_{(L-1)(L-1)}
\end{array}\right]
$$

Once the ZCT matrix generated subsequently it applied to each of the complex vectors to transform it into new vectors from which the one of the data will selected based on minimum PAPR values that will be used for transferring to receiver through wireless channel. The construction of modified complex vector can be obtained through the following equation

$$
\begin{gathered}
Y=A \cdot S_{t} \\
Y=[A] \cdot\left[S_{1}, S_{2}, \cdots S_{L-1}, S_{L}\right]^{T} \\
Y_{i, m}=\sum_{l=1}^{L} a_{m, l} \cdot S_{t, l} m=1,2, \ldots, L \text { and } t=1,2, \ldots, T
\end{gathered}
$$


Where $a_{m, l}$ represents the $m^{\text {th }}$ row and $l^{\text {th }}$ column of precoder matrix.

$$
Y_{i, m}=\sum_{l=1}^{L}\left(e^{j \frac{\pi(m L+l)^{2}}{L^{2}}}\right) \cdot S_{t, l}
$$

The complex baseband ZCMT vectors are subjected to IFFT for each antenna with N number of subcarriers can be obtained as

$$
S_{i, n}=\frac{1}{\sqrt{L}} \sum_{m=1}^{L} Y_{i, m} e^{j 2 \pi \frac{n}{L} m} n=1,2, \ldots, L
$$

The calculation of PAPR of proposed methodology of each signal can be calculated as

$$
P A P R=\frac{\max \left[\left|S_{i, n}\right|^{2}\right]}{E\left[\left|S_{i, n}\right|^{2}\right]}
$$

\subsection{Transmitting Signal Through Channel}

The channel with $\mathrm{N}$ outputs and $\mathrm{M}$ inputs is denoted as a $\mathrm{M} \times \mathrm{N}$ matrix: where each entry hij denotes the attenuation and phase shift (transfer function) between the I th transmitter and the $\mathrm{j}$ th receiver

$$
A=\left[\begin{array}{ccc}
h_{11} & \cdots & h_{1 N} \\
\vdots & \ddots & \vdots \\
h_{M 1} & \cdots & h_{M N}
\end{array}\right]
$$

The MIMO signal model is described as $\vec{r}=H \vec{S}+\vec{n}$, where $r$ is channel matrix of size $M \times N, r$ is the transmitted vector of size $M \times 1$, and $n$ is the noise vector of size $N \times 1$.

Receiver side

\subsection{Channel Estimation}

Kalman Filter is used for tracking MIMO channels based on a low order autoregressive (AR) model. Estimation procedure: The Kalman filter estimates the state. $x \in R n$ of a discrete-time controlled process that is governed by the linear stochastic difference equation

$$
\mathrm{T} s_{k}=A x_{k-1}+B u_{k-1}+w_{k-1}
$$

with a measurement $z C R m$ that is

$$
z_{k}=c H \mathrm{~T} s_{k}+n_{k}
$$

The random variables $w_{k}$ and $u_{k}$ represent the process and noise respectively. They are assumed to be independent of each other, and with normal probability distributions.

$$
\begin{aligned}
& p(w) \approx N(0, Q) \\
& p(u) \approx N(0, R)
\end{aligned}
$$

The $\mathrm{n} \times \mathrm{n}$ matrix $\mathrm{A}$ in the difference equation relates the state at the previous time step $\mathrm{k}-1$ to the state $\mathrm{k}$ at the current step. The $\mathrm{n} \times 1$ matrix $\mathrm{B}$ relates the optional control input $\mathrm{u} C \mathrm{R} 1$ to the state $\mathrm{x}$.

The state estimate at step $\mathrm{k}$ is defined as $\hat{\mathrm{T}} s_{k}$ belongs to real number by given knowledge of the process prior to step $k$, and that to be $a$ post-prior state estimate at step $k$ given measurement $z_{k} \cdot a$ priori and a posteriori estimate errors are

$$
\begin{aligned}
& \bar{e}=R s_{k}-\hat{R} s_{k} \\
& \bar{e}=R s_{k}-\hat{R} s_{k}
\end{aligned}
$$


The prior estimate error covariance is then

$$
\bar{P}_{k}=E\left[\begin{array}{c}
-\bar{T} \\
e_{k} e_{k}
\end{array}\right]
$$

and the post-prior estimate error covariance is

$$
P_{k}=E\left[e_{k} e_{k}^{T}\right]
$$

A state estimate $\hat{R}$ can be computed as a linear combination of an a priori estimate $\hat{R}_{k}$ and a weighted difference between an actual measurement $z_{k}$ and a measurement prediction ${ }^{\wedge} \mathrm{cH} R \mathrm{R} k$

$$
\hat{R}_{k}=\hat{R} s_{\bar{k}}^{-}+K\left(z_{k}-c H \hat{R s_{\bar{k}}}\right)
$$

The difference $z_{k}-c H \hat{R s_{-}}$is called the measurement innovation, or the residual. The $n \times m$ matrix $K$ is chosen to be the gain or blending factor that minimizes the posterior error covariance.

$$
K_{k}=\bar{P}_{K} H^{T}\left(c H \bar{P}_{K} c H^{T}+R\right)^{-1}
$$

After each time and measurement update pair, the process is repeated with the previous a posteriori estimates used to project or predict the new a prior estimates [11], [12], [14].

\subsection{System Model for STBC Coded MIMO}

The state space equations for tracking the MIMO channel can be expressed as:

$$
\begin{aligned}
& \operatorname{ch}(t+1)=A(t) \operatorname{ch}(t) \\
& \mathrm{T} s_{k}=C(t) \operatorname{ch}(t)+u(t)
\end{aligned}
$$

where $h$ is the channel tap, $A$ is a time-varying transition matrix, $C$ is the observation matrix and $u$ is the measurement noise vector. On the receive antenna, the noise $n$ has the Gaussian probability density function with

$$
p(n)=1 / \sqrt{2 \prod \sigma^{2}} \exp \left[-\left(n-\mu^{2}\right)\right] / 2 \sigma^{2}
$$

with $\mu_{h i j}=0$ and $\sigma_{h^{2} i j}=N o / 2$. A first-order Auto-Regressive (AR) model provides a sufficient model for time varying channels. Therefore, $A$ can be a diagonal matrix of autoregressive model factor $\alpha$, where

$$
\alpha=E\left[\operatorname{ch}_{i j}(t+1) * \operatorname{ch}^{*}{ }_{i j}(t)\right]
$$

The Extended Kalman filter equations for MIMO channel are divided into two parts. First part is the predictor:

$$
\begin{aligned}
& \tilde{c h}(t+1 \mid t)=A(t) \tilde{c h}(t \mid t) \\
& P(t+1 \mid t)=A(t) P(t \mid t) A^{* T}(t) \\
& \varepsilon(t)=\mathrm{T} s(t)-C(t) \tilde{c h}(t+1 \mid t)
\end{aligned}
$$

And the second part is the update:

$$
\tilde{c h}(t+1 \mid t+1)=\tilde{c h}(t+1 \mid t)+K(t) \varepsilon(t)
$$

Where $R_{u}=\beta I$ and $\beta$ is a covariance of the noise vector $\mathrm{v}$. The $K$ matrix is called the Kalman gain and the $P$ matrix is called the estimation error covariance [12], [15]. 


\subsection{Maximum Likelihood}

For the received signal $s(t)$, the decoder selects the code vector $s(t)$ is given in the following equation (33)

$$
\hat{\mathrm{T} s}(t)=\underset{s}{\arg \min }(\|R s(t)-c H(t) \mathrm{T} s(t)\|)^{2}
$$

Where Euclidean normalization function is denoted as $\|\cdot\|$. The search is done with the overall possible code vectors Ts and with the intention of decode the Ts $(t)$ from $R s(t)$, the decision statistic vector $z(n)=\tilde{c} h(t+1 \mid t+1)+c H^{H}(t) R s(t)$ is formed. Once the Estimated formulated signal using MLE then it will compared with transmitted signal to calculate BER in terms of SNR.

\section{RESULT AND DISCUSSION}

In wireless communication, channel estimation is the need to enhance the reliability and performance of the system. Here we have calculated the two parameters bit error rate (BER) in terms of signal to noise ratio (SNR) (Eb/No (dB)) and channel capacity for various number of signals. We compare the performance of our proposed methodology with the "Known Channel", "Unknown channel", "Conventional OSTBC Theory", "EKF-AMLE [15]"

\subsection{Performance Evaluation Based on BER}

The following figure 1 represents the comparative analysis of BER of various methodologies. By analyzing the BER values, our proposed algorithm performed better than the EKF-AMLE and Unknown channel and our proposed algorithm achieves very closer result with the Known channel and OSTBC theory result. In our previous work, we does not concentrate on PAPR issues obtained in MIMO-OFDM and in the receiver side, $\mathrm{N}$ number of antennas are used to reduce the BER rate. The $\mathrm{N}$ number of receiver antenna will violate the cost effectiveness. With the intention of reducing the number of receiver antenna and PAPR, in our proposed methodology, we incorporate the ZCT for reducing PAPR and the combination of OSTBC and EKF helps reducing the $\mathrm{N}$ number of received antenna for cost effectiveness. Our proposed methodology does not violates the BER values moreover; it improves the performance of BER compared with our previous methodology (EKFAMLE) which is stated in the figure 1. The correlation between the proposed BER and the BER result obtained with the Known channel are closed together which is described in the figure 2.

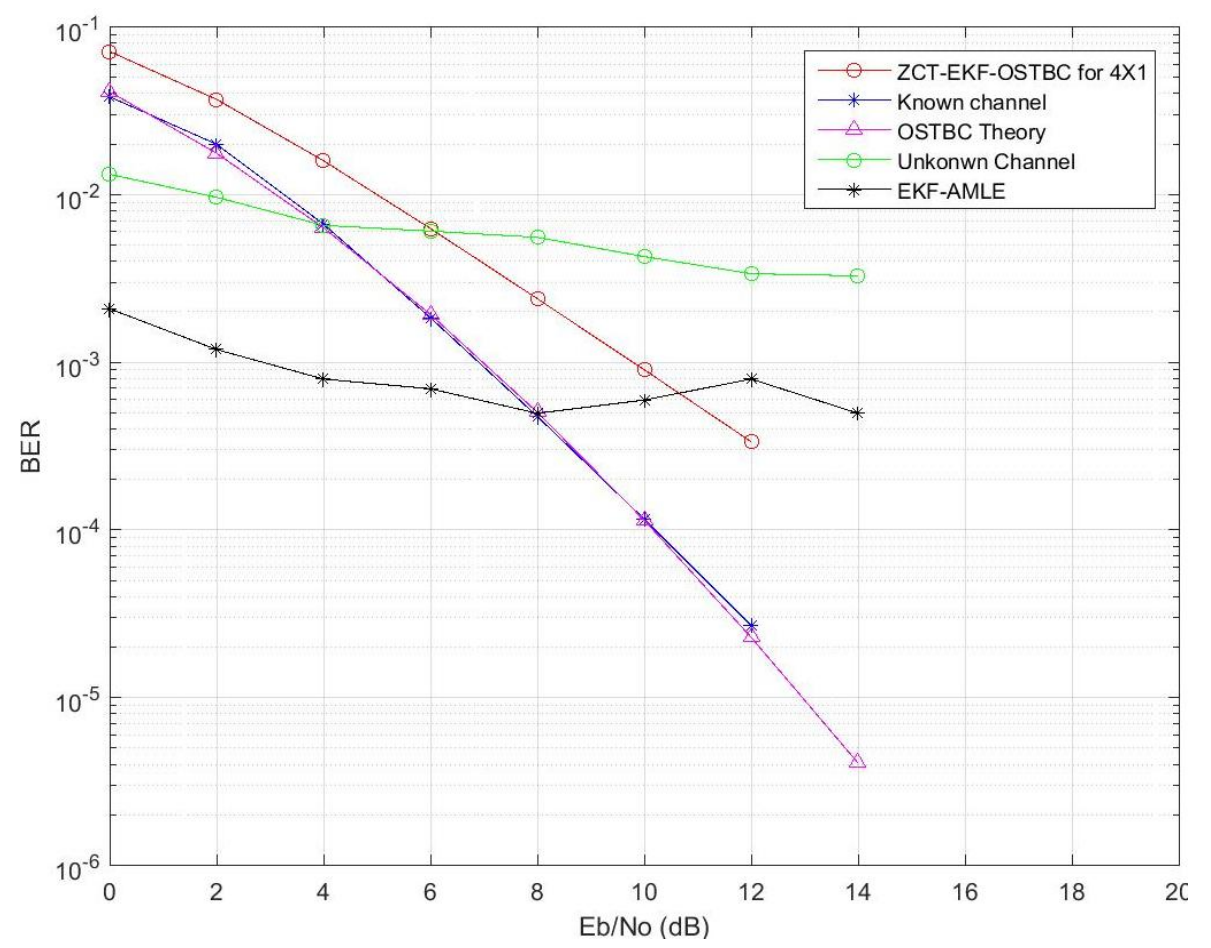

Figure1. Depicts that the comparative analysis of BER values of various methodologies 


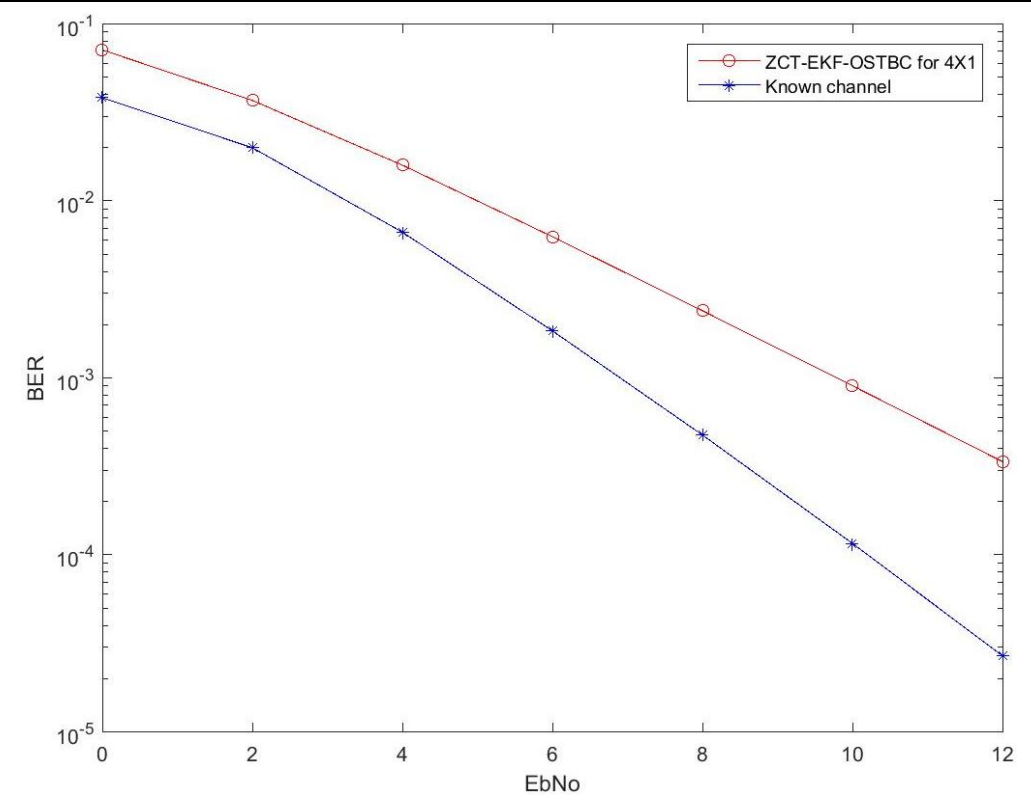

Figure2. Describes that the comparative analysis of BER values of proposed methodology with the known channel

\subsection{Comparative Analysis of PAPR}

In this section, we evaluate the various methodology to get the proof of effectiveness of our proposed methodology in terms of PAPR values. For that, in this section we selected our previous methodology EKF-AMLE [15]. With the intention of represent the effectiveness of ZCT algorithm we present a methodology without ZCT algorithm, which is stated as EKF-OSTBC. The PAPR values are obtained from each methodologies such as EKF-AMLE, EKF-OSTBC and ZCT-EKF-OSTBC for various values of complementary cumulative distribution function (CCDF). The comparative analysis of PAPR is represented in the following figure 3. By analysing the figure 3, EKF-OSTBC algorithm provides high PAPR values than the other two algorithm the reason behind this is using of OSTBC coding methodology added with OFDM in transmitter side. However, using of OSTBC methodology helps to reduce the number of antennas but it affects the PAPR values since ZCT algorithm induced with EKF-OSTBC with the intention of reducing the PAPR values. We proved that the obtained resultant values of ZCT- EKF-OSTBC values performed well than the EKF-AMLE algorithm, which represented in the following figure 3. The capacity of selected known channel for the wireless communication is presented in the above figure 4 .

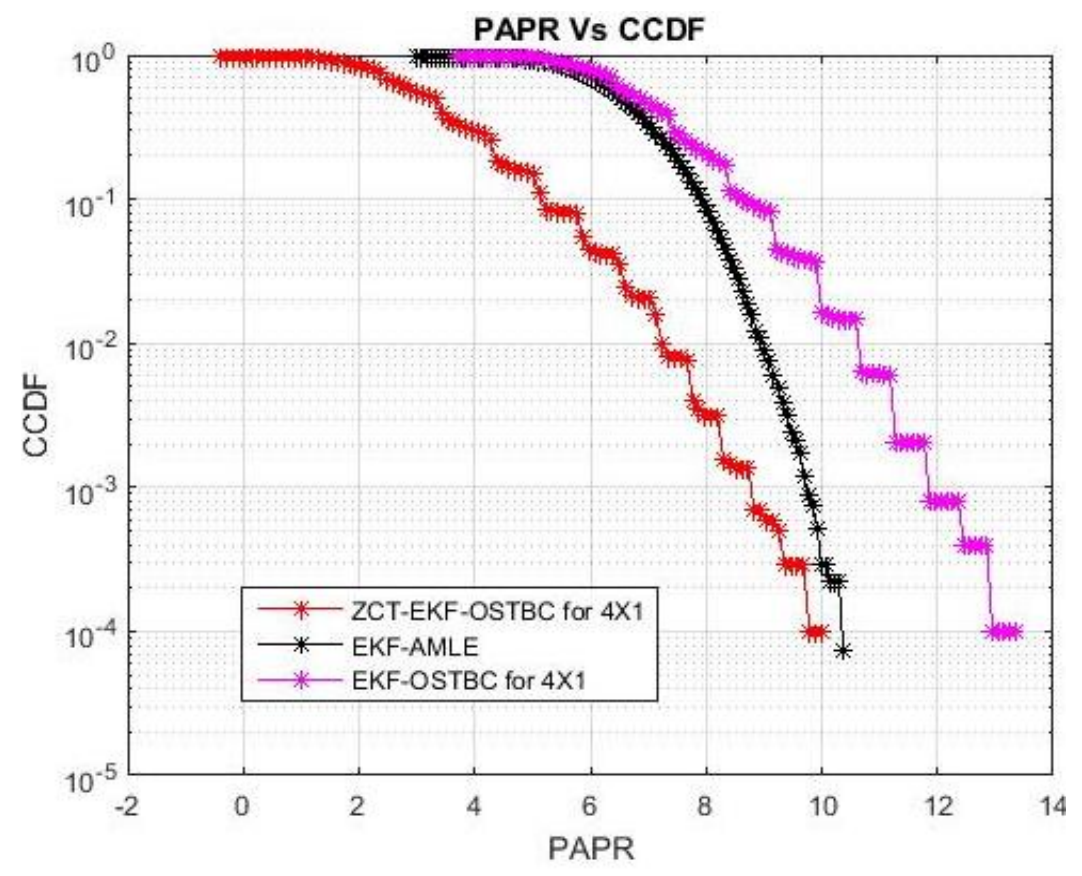

Figure3. Shows that the comparative analysis of PAPR 


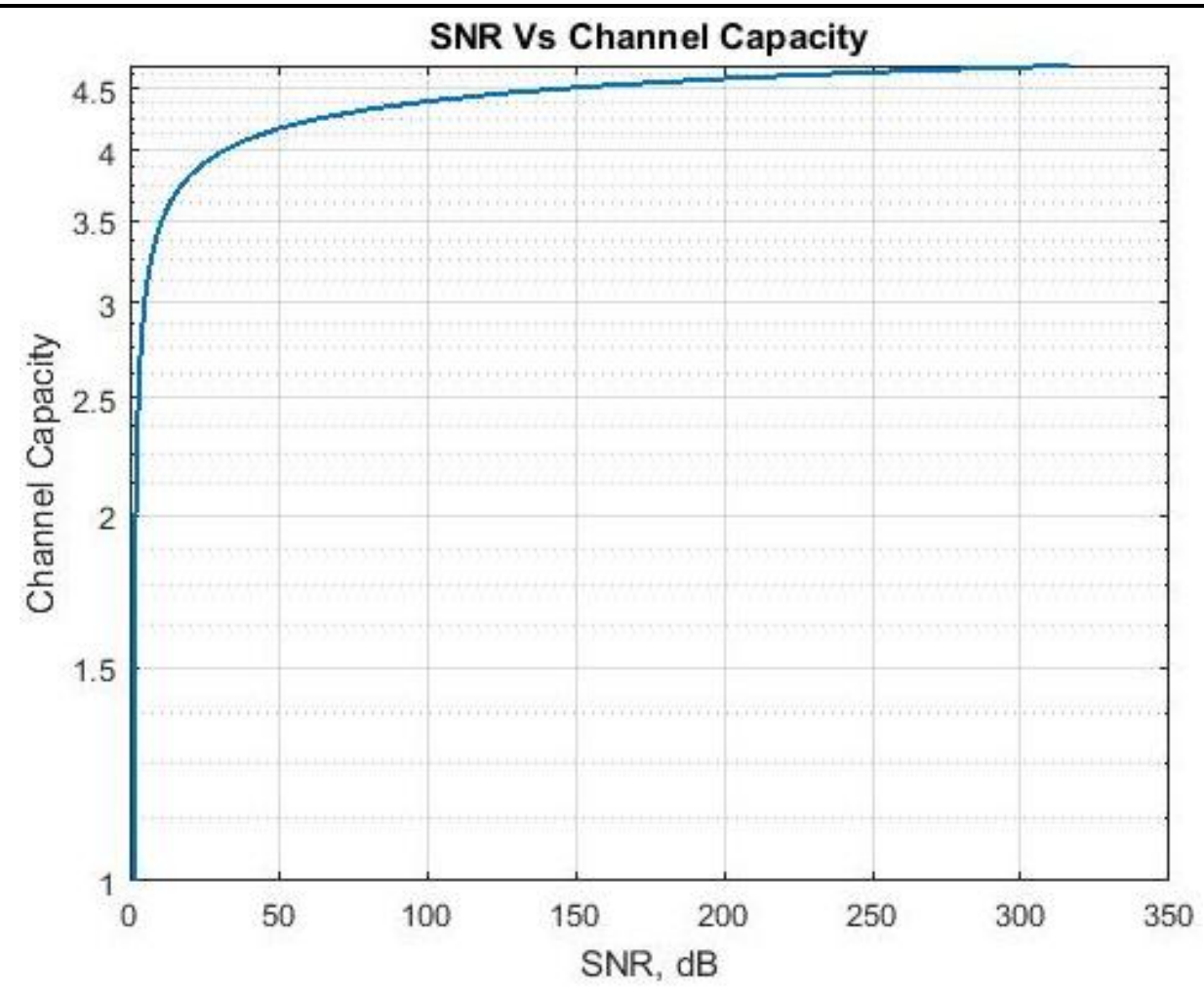

Figure4. Reveals the channel capacity of the proposed ZCT-EKF-OSTBC methodology

\section{Conclusion}

In this paper, we have analysed and investigated ZCT-OSTBC-EKF based MIMO-OFDM system to enhance the diversity and to reduce the PAPR values. The OSTBC methodology used for enhance the transmitter diversity gain also, it works well and produces less BER values. However, the additional coding methodology in the OFDM system is precedes to yield more PAPR during the transmission. We used ZCT methodology to reduce the PAPR values and the combination of ZCT-OSTBC leads to improve the transmitter diversity of MIMO-OFDM system. Moreover, the receiver diversity is can be improved using of Extended Kalman Filter (EKF). By enhancing the transmitter diversity and receiver diversity, we achieved the spatial diversity, which directs to reduce the BER values and PAPR values. Finally, we evaluated performance of our proposed ZCT-OSTBC-EKF methodology and it is performed well in terms of BER and PAPR by compared with the existing methodologies EKFAMLE.

\section{REFERENCES}

[1] Winters, J. H. (1987). On the capacity of radio communications systems with diversity in a Rayleigh fading environment. IEEE Journal on Selected Areas Communications, 5(5), 871-878.

[2] Bingham, J. A. C. (1990). Multicarrier modulation for data transmission: An idea whose time has come. IEEE Communications Magazine, 28(5), 5-14

[3] Jing-Jyun You and Shyue-Win Wei, "Performance Verification of Successive Interference Cancellation for STBC MIMO-OFDM System with Channel Correlation", Wireless Personal Communications, Vol. 50, No. 4, pp. 529-543, 2009.

[4] Xiaodong Zhu, "A Low-BER Clipping Scheme for PAPR Reduction in STBC MIMO-OFDM Systems", Wireless Personal Communications, Vol. 65, No. 2, pp. 335-346, 2012.

[5] G Wunder, RFH Fischer, H Boche, S Litsyn, J-S No, The PAPR problem in OFDM transmission: new directions for a long-lasting problem. IEEE Signal Proc. Mag.30(6), 130-140 (2013)

[6] E Costa, M Midrio, S Pupolin, Impact of amplifier nonlinearities on OFDM transmission system performance. IEEE Commun. Lett.3(2), 37-39 (1999)

[7] Mouna Sghaier, Fatma Abdelkefi, and Mohamed Siala, "PAPR reduction scheme with efficient embedded signaling in MIMO-OFDM systems", EURASIP Journal on Wireless Communications and Networking, Vol. 240, pp. 1-16, 2015. 
[8] S. H. Han and J.H.Lee, "PAPR Reduction of OFDM Signals using a Reduced Complexity PTS Technique”, IEEE Trans. on Signal Processing Letters, vol.11, no.11 pp. 887-890, November 2004.

[9] C.L.Wang and Y. Ouyang, "A Low-Complexity selected mapping schemes for Peak-to- Average Power Ratio Reduction Technique for OFDM systems", IEEE Trans. on Signal Processing, vol. 53 , no.12, pp. 4652 - 4660, December 2005.

[10] D. Guel and J. Palicot, "FFT/IFFT Pair based Digital Filtering for the Transformation of Adding Signal PAPR Reduction Techniques in Tone Reservation Techniques", Fifth International Conference onWireless and Mobile communications, pp. 200-204, August 2009.

[11] I. Baig and V.Jeoti," PAPR Reduction in OFDM Systems: ZadoffChu Matrix Transform Based Pre/Post-Coding Techniques", IEEE Trans. on Communication Systems and Networks, pp. 373377, July 2010.

[12] Tincy Mary Mathew, Anirudh Mudaliar, "Alamouti STBC MIMO OFDM System using ZCT Precoded Based SLM Technique for PAPR Reduction", International Journal of Science, Engineering and Technology Research, Vol. 3, No. 9, pp. 2363-2369, 2014.

[13] Imran Baig, Varun Jeoti and Micheal Drieberg, "A ZCMT Precoding Based STBC MIMOOFDM System with Reduced PAPR", In proceedings of National Postgraduate Conference (NPC), pp. 1-5, Kuala Lumpur, 2011.

[14] Javaid A. Sheikh, Shabir A. Parah, G. Mohiuddin Bhat, Mehboob ul Amin, "PAPR reduction in MIMO-OFDM System using combination of OSTBC Encoder and Spreading Code Sequence", International Conference on Multimedia, Signal Processing and Communication Technologies, pp. 11-15, Aligarh, 2013.

[15] Sanjay Kumar K V S and B L Malleswari, "Channel Estimation Using Extended Kalman Filter and Adaptive Maximum Likelihood Estimator in MIMO-OFDM System", International Journal Of Applied Engineering Research, Vol. 10, No. 12, pp. 30861-30882, 2015. 